\title{
Impact of the ENDF/B-VIII.0 library on modeling nuclear tools for oil exploration
}

\author{
Marie-Laure M auborgne ${ }^{1, *}$, R. J. Radtke ${ }^{1}$, Christian Stoller ${ }^{1}$, and Fabien Haranger $^{1}$ \\ ${ }^{1}$ Schlumberger, Schlumberger Houston Formation Evaluation Integration Center, Sugar Land, TX, USA
}

\begin{abstract}
In the oil field, exploration of the subsurface through well logging provides measurements of the characteristics of rock formations and fluids to help identify and evaluate potential reservoirs. Downhole nuclear measurements focus on formation properties such as natural radioactivity, formation density, and hydrogen content, as well as the identification of the elemental and mineralogical composition of the rock through spectroscopy. Accurate nuclear modeling is a fundamental part of nuclear well logging tool development, from concept through design to response characterization. Underlying the accuracy of nuclear modeling is a good knowledge of nuclear cross sections of the elements in the tool, borehole, and subsurface formations. The recent focus on replacing tools based on radio-isotopic sources with those based on D-T neutron generators opens many opportunities for new measurements but highlights the deficiencies of current cross sections. For example, in neutron-induced inelastic and capture gamma ray spectroscopy, major obstacles come from a lack of or inaccuracies in the cross sections of essential materials.
\end{abstract}

\section{Introduction}

In the oilfield industry, exploration of the subsurface is essential to answer questions regarding location, type, and quantity of hydrocarbon as well as its producibility. Well logging provides measurements of the rock formation characteristics and of the fluids present in the formation's pore spaces. The main nuclear measurements in oil field use are natural radioactivity, density, neutron porosity, and gamma ray spectroscopy.

The downhole drilling environment can be severe: these tools must work at temperatures that may exceed $175^{\circ} \mathrm{C}$, are exposed to repeated shocks of $100 \mathrm{~g}$ or more, and must be protected by housings withstanding downhole pressures of $2000 \mathrm{~atm}$ or more. In these difficult environments, delivering accurate and reliable measurements is a challenge [1].

With the introduction of pulsed neutron generators, new measurements have been developed such as capture and inelastic gamma ray spectroscopy, which directly measure elements in the formation including carbon. The complex measurements and the introduction of machine learning increase the need for accurate modeling [2][3]. Major modeling uncertainties come from the cross sections. The latest major release of the evaluated nuclear reaction data (ENDF) library [4] has major changes in neutron reaction cross sections for isotopes of interest in well logging.

\section{Context}

Modeling is widely used when designing a tool to help find the best compromise between mechanical constraints - ensuring tool integrity under shock, vibration, and temperature-and measurement quality. We traditionally use the MCNP transport code [5].

Once a tool has been designed and built, the modeling is benchmarked against laboratory measurements. At this point, modeling is used to expand, complement, and refine the experimental database, which is the foundation of response algorithm development. This enables tool performance to be predicted more accurately, facilitating the development of better answer products. In the laboratory, only a limited number of actual or simulated rock formations are available. Many formations such as anhydrite or gasfilled formations are, at best, difficult to obtain in a laboratory environment. In addition, for practical and financial reasons, only a small number of rock formations with different properties, such as porosity, lithology, borehole size, and formation fluid, are available.

Logging measurements introduce certain limitations and challenges, such as the noise (background) signal generated by the tool itself and limitations on the acquired data. Despite these limitations, it has been observed that many discrepancies between measurement and simulation are caused by deficiencies in the modeling and in particular by the lack of accurate gamma ray production cross sections.

We know that neutron-capture $\gamma$-ray line data are missing since ENDF/B-VII, which split the natural compound into individual isotopes [2][6]. In ENDF/BVIII.0 new and improved neutron-capture $\gamma$-ray lines for ${ }^{23} \mathrm{Na},{ }^{27} \mathrm{Al},{ }^{28} \mathrm{Si}$, and ${ }^{35} \mathrm{Cl}$ and ${ }^{37} \mathrm{Cl}$ have been included [6].

\footnotetext{
Corresponding author: M M auborgne@slb.com
} 


\section{Tool description}

The results presented in this paper are based on a recently introduced geochemical logging tool [7]. A pulsed neutron generator (PNG) irradiates the formation surrounding the tool, and the induced gamma rays are detected with a $\mathrm{LaBr}_{3}: \mathrm{Ce}$ scintillator. The neutron pulsing scheme and time-gating of the acquired data enable clean separation of capture and inelastic gamma ray spectra. Analysis of these spectra produces dry weight fractions of elements and minerals in the formation. The combination of capture and inelastic spectroscopy can be used to quantify the amount of total organic carbon in the formation, providing direct information on oil reserves.

The PNG, photomultiplier tube (PMT), and associated electronics were designed and manufactured in-house. Many of these components have applications beyond the oil patch. Similar in-house PMTs are used in space exploration; for example, they can be found in the Hubble Space Telescope [8]. Additionally, there is ongoing collaboration with NASA to adapt the PNGs to planetary exploration on Venus [9], Titan [10] and the moon.

The tool has been tested extensively in quarried and laboratory formations designed specifically for geochemical logging applications (Fig. 1). In such a controlled environment, high-quality measurements can be made with much longer acquisition times than during commercial use (a few hours compared to minutes or seconds). These experiments are the primary source of data used to define the interpretation algorithms.

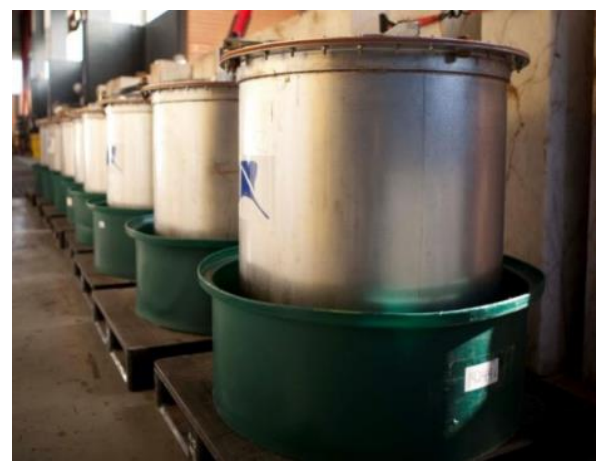

Fig. 1: Some of the laboratory formations used for tool characterization

The key step in interpreting the measurements is the analysis of the acquired spectra. We record two different energy spectra, one during the neutron burst of the PNG, when $\gamma$-rays are mainly produced by inelastic reactions, and a second one starting after the end of the burst, when the $\gamma$-rays come exclusively from capture reactions after correction for the gamma rays from natural background and neutron activation. The spectra are then decomposed into the contributions from different elements based on elemental standard spectra.

The standard spectra are derived by taking differences between spectra from a variety of targeted measurements. Modeled spectra are used to assist in the proper determination of the standards but are generally not accurate enough to be used in the spectral fitting algorithm. As will be seen, much of this inaccuracy follows from the cross-section evaluations.

The modeled standard spectra presented in this paper are computed using the Monte Carlo N-Particle (MCNP) transport code [5]. The code has been modified to allow the recording of gamma rays by the isotope and nuclear reaction that created them and by the spatial region where they were produced. The photon current entering the scintillator is converted to a pulse height spectrum using an MCNP-generated response map and convolution with an energy-dependent Gaussian function designed to replicate the measured spectrum and its spectral resolution.

\section{Test of the new cross sections}

For the spectroscopy measurement, we are only interested in $\gamma$-rays with an energy larger than $0.8 \mathrm{MeV}$. At very low energies $(<200 \mathrm{keV})$ modeling and experiment show poor agreement due to electronic threshold effects for small signals. The energy spectra are normalized to the integral above $600 \mathrm{keV}$ to exclude differences caused by the electronic threshold.

When comparing cross-section libraries, we only change the library for the studied element in the material corresponding to the sample in the input file. We focus only on the gamma-ray spectral shape as the absolute amplitude of neutron capture and inelastic cross sections are of secondary importance for this application.

\subsection{Validation on fresh water}

The signal coming from the tool is considered as noise, but for benchmarking and for the spectral processing, we must be able to reproduce it as accurately as possible. To validate the tool signal, we use a measurement taken in a very large water tank. For capture, the only significant contributor is the $2.2 \mathrm{-MeV}$ gamma ray of hydrogen. The burst benchmarking is more complex as we have the residual capture signal and some signal coming from inelastic reactions with oxygen.

Comparison of experimental results with modeling results is shown in Fig. 2. We have an excellent reproduction of the signal between 0.5 and $2.2 \mathrm{MeV}$. The reproduction of the tool signal (above $2.2 \mathrm{MeV}$ ) is demanding and we overestimate it above $7 \mathrm{MeV}$. ENDF/B-VIII.0 introduced no changes to the hydrogen capture $\gamma$-ray energy spectrum.

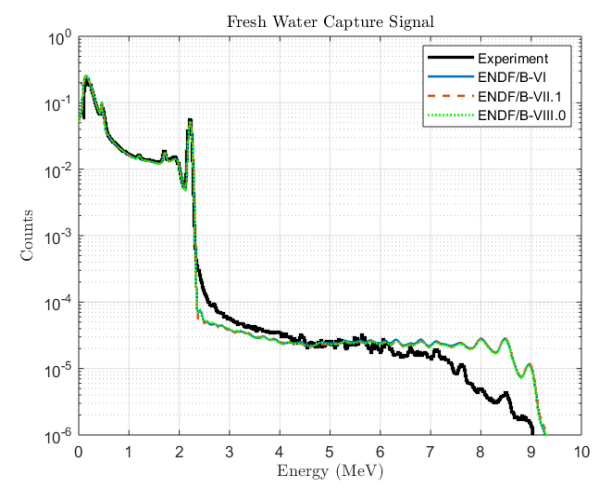




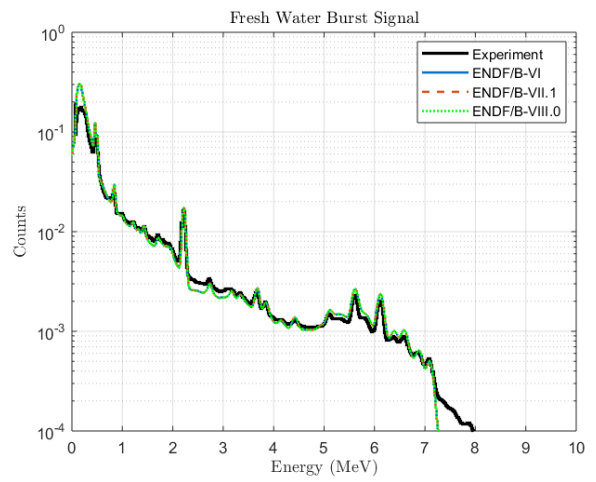

Fig. 2: Energy spectra recorded in the water tank compared with modeled spectra during capture and burst

\subsection{Silicon}

To obtain the spectroscopy standards, we generally use formations composed of a mix of quartz $\left(\mathrm{SiO}_{2}\right)$ and the desired element. This has the advantage of reducing the weight of the needed element (which must be large enough to represent an infinite medium around the tool) and providing a direct measurement of the sensitivity to the desired element compared to the silicon reference element in a given environment.

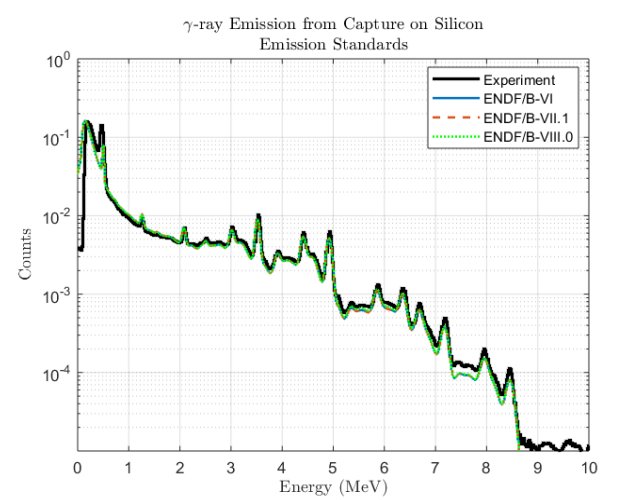

Fig. 3: Comparison of capture $\gamma$-ray spectra from silicon from experiment and modeling with various libraries

The agreement between the modeled and experimental capture silicon standards is very good (Fig. 3). We can spot a small difference at about $6 \mathrm{MeV}$ between ENDF/B-VIII.0 and previous versions. This is linked to an increase of the strength of the $6.047 \mathrm{MeV}$ line. This line and some others are not listed in [11], but we can find them in other work [12]. Silicon primary $\gamma$ ray line data have been updated in ENDF/B-VIII.0 [4].

The gamma ray spectra from inelastic reactions show larger differences (Fig. 4). By looking at the raw (not processed with detector response) modeling results in Fig. 5, we can see that the shape of the spectrum is better defined for ENDF/B-VII and above and corresponds to the main lines measured in [13], which is limited in energy range by the use of a fast neutron reactor. But the relative contribution of the high-energy part for ENDF/B-VII and above is lower than our observations.

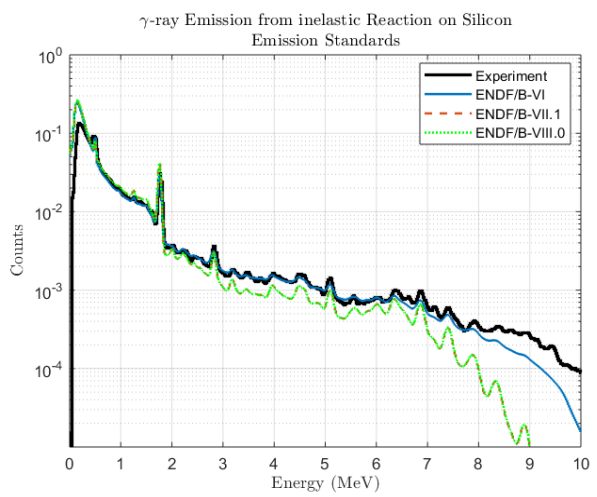

Fig. 4: Comparison of inelastic $\gamma$-ray spectra from silicon from experiment and modeling with various libraries

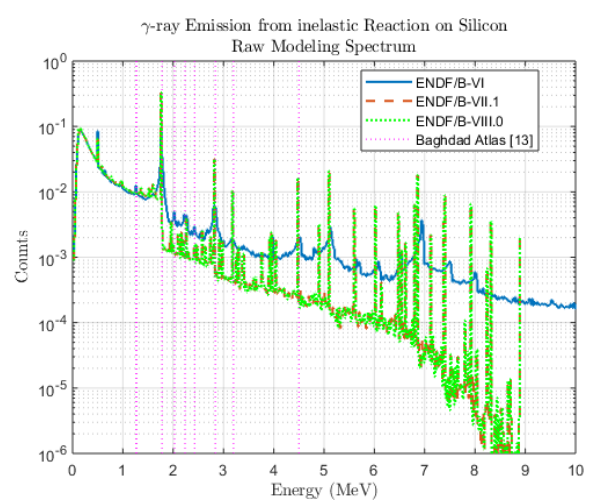

Fig. 5: Comparison of raw inelastic $\gamma$-ray spectra from silicon modeled with various libraries

For silicon, we will continue using the ENDF/B-VI.2 library in general. For a dedicated capture study, the ENDF/B-VIII.0 library brings small improvements.

\subsection{Iron}

The ENDF/B-VIII.0 cross-section library contains a reevaluation of iron cross sections based on CIELO collaboration findings [14]. Iron is of particular interest to us not only because our tools are mainly built out of steel but also because shale can have a high concentration of iron.

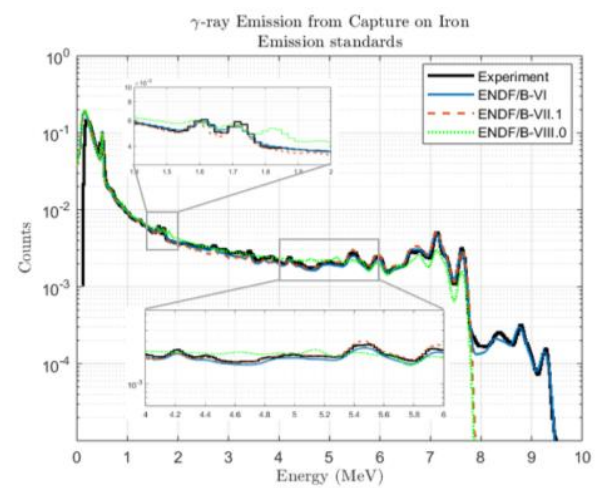

Fig. 6: Comparison of capture $\gamma$-ray spectra from iron from experiment and modeling with various libraries

Fig. 6 shows the comparison between the capture iron signal and the modeling with various cross-section libraries. ENDF/B-VI and VII agree nicely up to $8 \mathrm{MeV}$, but ENDF/B-VIII.0 shows some strange features. The gamma ray emission spectrum changes significantly 
depending on the library choice as shown in Fig. 7. Many capture gamma rays present in the IAEA database [11] are missing in the ENDF/B-VIII.0 version and several were added.

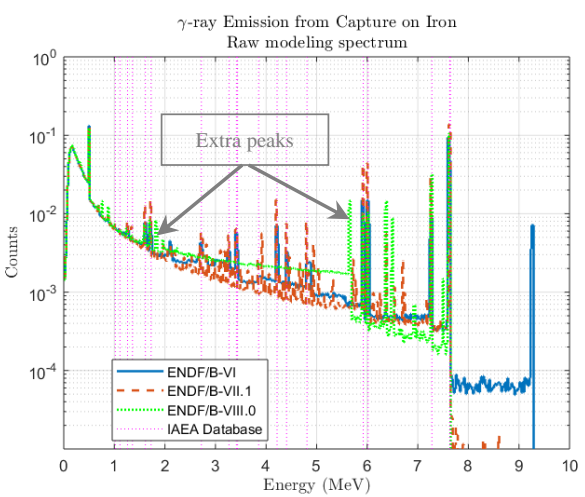

Fig. 7: Comparison of raw capture $\gamma$-ray spectra from iron modeled with various libraries

As for capture, we have a better agreement between modeling and experiment from ENDF/VII.1 than ENDF/B-VIII.0 for inelastic reactions (Fig. 8). For iron, we will continue using ENDF/B-VII.1 library.

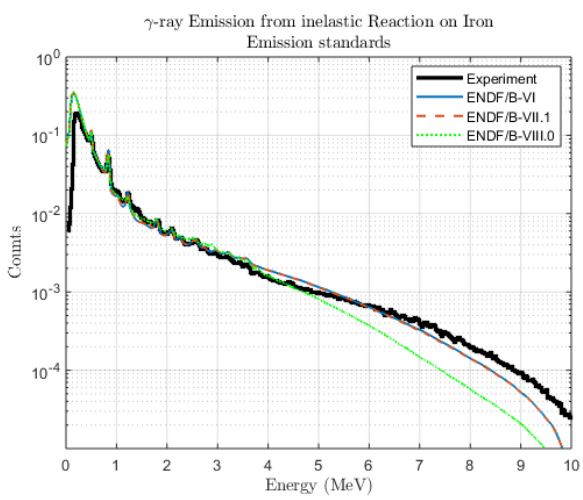

Fig. 8: Comparison of the iron inelastic $\gamma$-ray spectra from experiment and modeled with various libraries

\subsection{Manganese}

Another important element is manganese; even if it is rarely found in subsurface formations, it is often found in the alloys used in our tools. Being able to separate the signals coming from our tools from the signals coming from the formations we are investigating is crucial. Therefore, a good reproduction of the gamma ray signature of the tool is essential. Manganese has a larger thermal neutron absorption cross section than iron (13.36 b vs $2.59 \mathrm{~b}$ [11]), so it can have a large contribution to the tool signal.

To extract the manganese standard spectrum, we use a formation mix of quartz and manganese oxide.

Significant changes in modeling results are introduced by using the ENDF/B-VIII.0 cross-section library. Using previous versions, we were able to model the energy spectrum accurately (Fig. 9). By comparing the neutron capture gamma ray lines from the raw modeling we can see that a significant number of lines are missing (Fig. 10) in ENDF/B-VIII.0. We only display the main reference lines of the database for readability. In the latest version, it seems that no gammarays are emitted with an energy between 2 and $5 \mathrm{MeV}$. The strength of the gamma-ray emission has also been reduced at the very high end of the spectrum.

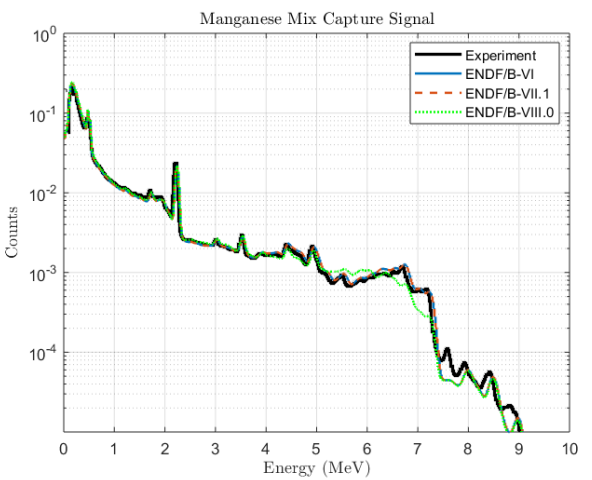

Fig. 9: Comparison of capture $\gamma$-ray spectra from the manganese mix from experiment and modeled with various libraries

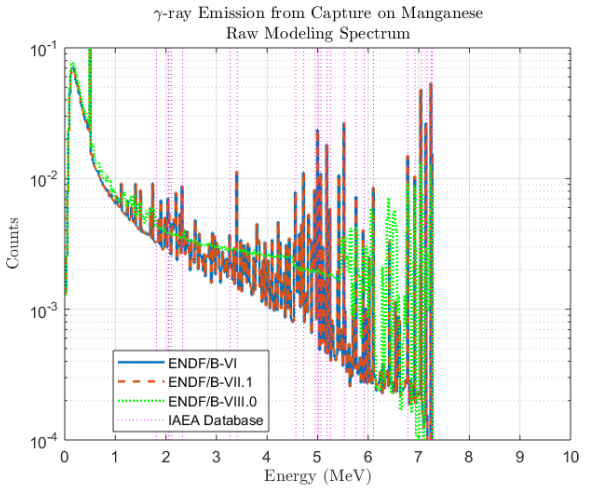

Fig. 10: Comparison of raw capture $\gamma$-ray spectra from manganese modeled with various libraries

Inelastic modeling results show smaller differences between the libraries, but we did not determine the spectra experimentally. We will continue using the ENDF/B-VII.1 library.

\subsection{Magnesium}

Magnesium is the key marker to differentiate between two types of rocks: limestone $\left(\mathrm{CaCO}_{3}\right)$ and dolomite $\left(\mathrm{CaMg}\left(\mathrm{CO}_{3}\right)_{2}\right)$. But the thermal capture cross section is about 10 times lower for magnesium compared with calcium (53.7 mb vs. $415 \mathrm{mb}$ [11]). In dolomite, calcium gamma rays dominate. That is why we use a magnesium mixture to determine the magnesium standard.

The comparison of the detected capture gamma rays from magnesium between experiment and modeling in Fig. 11 is striking. There is a clear degradation between ENDF/B-VI and the later versions: the resolution is degraded, and the high-energy part of the spectrum is missing. Fig. 12 shows that all gamma ray emission lines above $1 \mathrm{MeV}$ in the IAEA database are present in the ENDF/B-VI evaluation (based on the natural isotopic composition of magnesium) but only a few, very broad lines are present in later versions (based on the isotopic composition). 


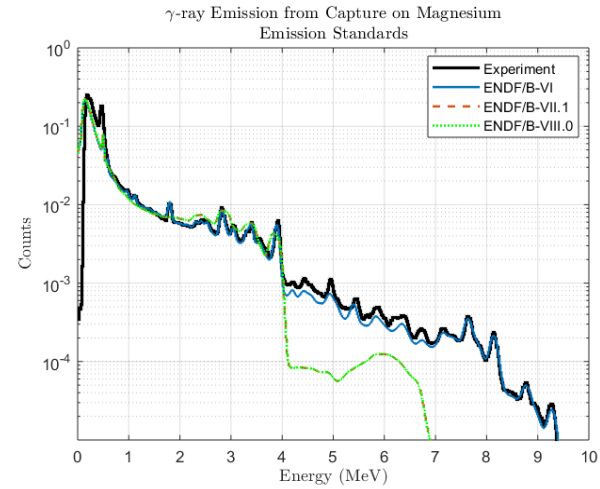

Fig. 11: Comparison of capture $\gamma$-ray spectra from magnesium from experiment and modeled with various libraries

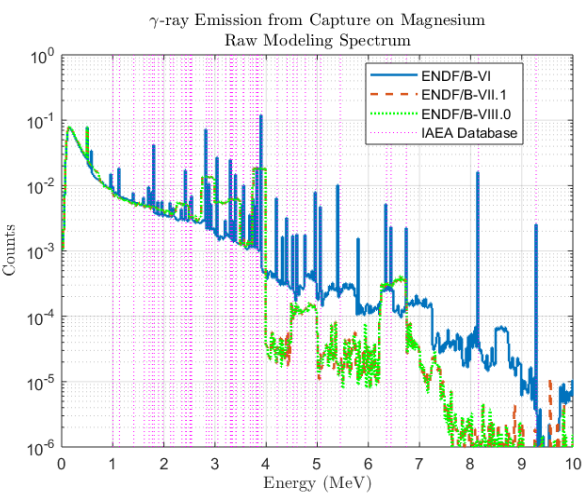

Fig. 12: Comparison of raw capture $\gamma$-ray spectra from magnesium modeled with various libraries

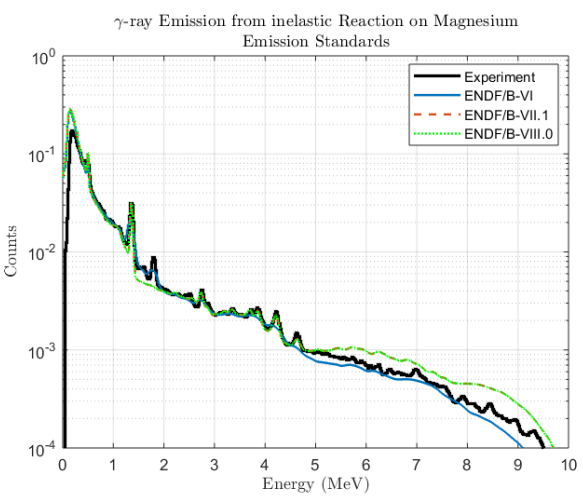

Fig. 13: Comparison of inelastic $\gamma$-ray spectra from magnesium from experiment and modeled with various libraries

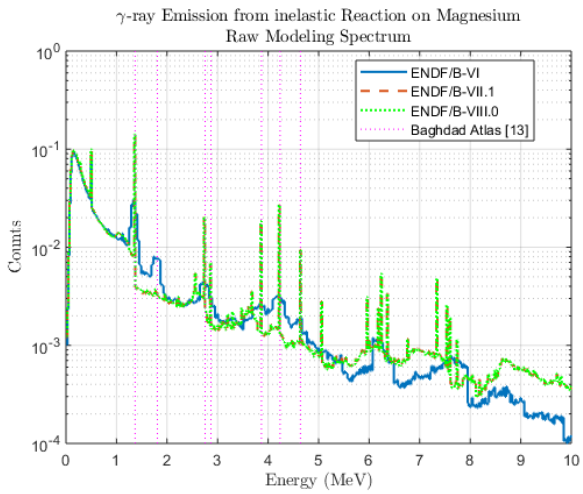

Fig. 14: Comparison of raw inelastic $\gamma$-ray spectra from magnesium modeled with various libraries and [13]

On the inelastic spectrum side, the peak at about $1.8 \mathrm{MeV}$ is well reproduced with the ENDF/B-VI library, but resolution is lost at higher energy (Fig. 13).
The $1.808-\mathrm{MeV}$ peak has already been seen in [13]. But the raw modeling spectra in Fig. 14 demonstrate the difference in the peak resolution with the different libraries. Except for that missing peak, the ENDF/BVIII.0 is a clear improvement for the inelastic neutron reaction gamma rays of magnesium. For magnesium, we will continue working with ENDF/B-VI.2.

\subsection{Titanium}

Titanium is another important element for our tools, as it is often used to package the detectors due to its high strength, low density, and low atomic number. Its contribution to the total spectrum could be high due to the proximity of the material to the scintillator. It is also found in clay minerals.

In Fig. 15 and Fig. 16, capture gamma ray emissions are improved between ENDF/B-VI.1 and ENDF/BVIII.0, with a better agreement with database and energy definition except for some lines (at $\sim 2.5,4.5$, and $5.5 \mathrm{MeV}$ ) not present in the IAEA database. Still the best agreement is obtained with ENDF/B-VI.2. The gamma ray strength above $6 \mathrm{MeV}$ is too low in the latest release and worse than in ENDF/B-VII.1.

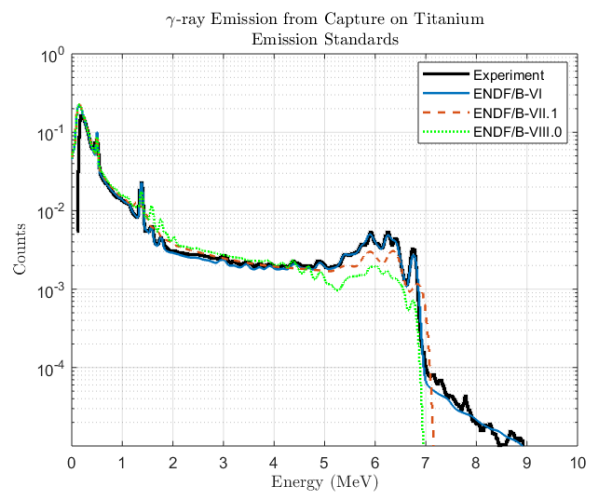

Fig. 15: Comparison of capture $\gamma$-ray spectra from titanium for experiment and modeled with various libraries

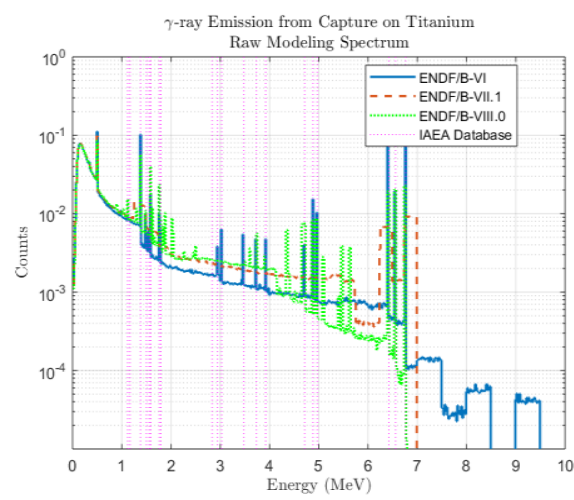

Fig. 16: Comparison of raw capture $\gamma$-ray spectra from titanium modeled with various libraries

We did not extract a gamma ray spectrum from the inelastic reaction on titanium, but modeled spectra with ENDF/B-VII.1 and ENDF/B-VIII.0. Energy lines seen in Fig. 17 agree with [13]. For titanium, we will continue working with ENDF/B-VI.2. 


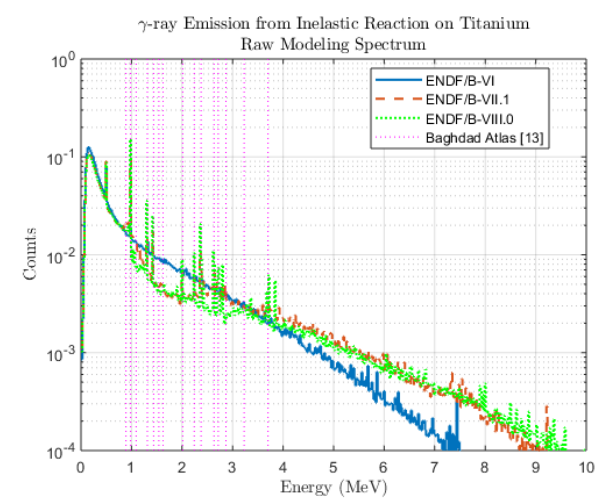

Fig. 17: Comparison of raw inelastic $\gamma$-ray spectra from titanium modeled with various libraries

\subsection{Sodium}

The water found underground is often saturated with salt $(\mathrm{NaCl})$, and the mud used during drilling may also be very saline. We checked the chlorine cross sections; only marginal differences were found. For sodium, the main differences in the spectra are between 4 and $6 \mathrm{MeV}$ (Fig. 18). Later releases improve accuracy compared with ENDF/B-VI. ENDF/B-VII.1 is more accurate in the 4- to $5-\mathrm{MeV}$ energy region by reducing the gamma ray emission probability.

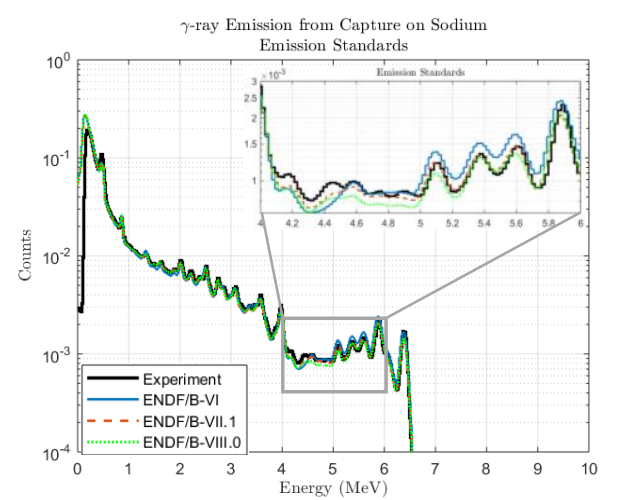

Fig. 18: Comparison of capture $\gamma$-ray spectra from sodium for experiment and modelling with various libraries

\subsection{Aluminum}

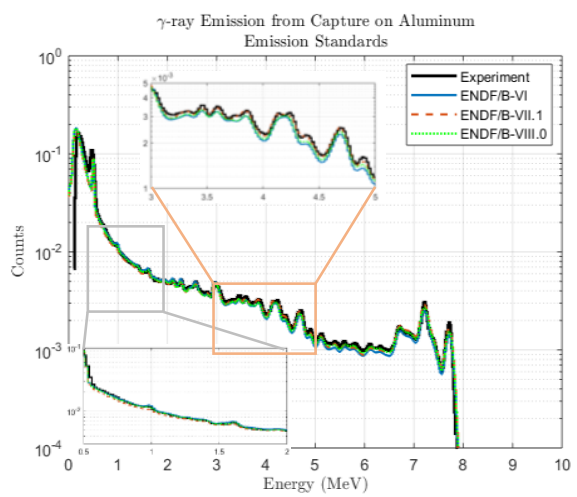

Fig. 19: Comparison of the capture $\gamma$-ray spectra from aluminum from experiment and modeled with various libraries

As for iron, aluminum is present both in our tool and in shales. The primary gamma emission has been modified in ENDF/B-VIII.0. We obtain a better match of our experimental results between 0.5 and $2 \mathrm{MeV}$ (Fig. 19); the higher strength of gamma ray emissions above $3 \mathrm{MeV}$ in ENDF/B-VII.1 improves the agreement. The difference between the libraries is minimal for aluminum.

\section{Conclusion}

We have seen a significant degradation in the accuracy of the secondary gamma ray energy distributions since the release of ENDF/B-VI. The biggest impact of the eighth release of the ENDF/B library on nuclear modeling for oil field is clearly in iron. There is a clear degradation in the accuracy of the capture gamma ray energy distribution between ENDF/B-VI.1 and ENDF/B-VIII.0 in iron and also in manganese and magnesium.

The titanium capture gamma ray energy distribution has been improved between release VII and VIII, but it is still not as accurate as in ENDF/B-VI.

For sodium and aluminum, differences between the releases are minimal, with a slightly better accuracy when using ENDF/B-VII.1.

\section{References}

1. M.-L. Mauborgne, et al., EPJ Web of Conferences $\underline{146,09009 \text { (2017) }}$

2. M.-L. Mauborgne, et al., EPJ Web of Conferences $\underline{\mathbf{1 4 6}, 09036(2017)}$

3. Okamoto, K. (ed.); International Nuclear Data Committee; Jan 1984; p. 57-74; IAEA consultants' meeting on nuclear data for bore-hole and bulkmedia assay using nuclear techniques; Cracow (Poland); 14-18 Nov 1983

4. D. A. Brown, et al., Nuclear Data Sheets, 148, pp. 1-142 (2018)

5. C. J. Werner, report LA-UR-17-29981 (2017)

6. A. M. Hurst, et al., EPJ Web of conference, 146. $09008(2017)$

7. R. J. Radtke, et al., SPWLA 53rd Annual Logging Symposium (2012)

8. J. L. Groves, et al., Oilfield Review, 18 (2006)

9. A. M. Parsons et al., IEEE (NSS/MIC/RTSD) (2016) DOI : 10.1109/NSSMIC.2016. 8069877

10. A. M. Parsons et al, AGU Fall meeting, 2018, P52C-08

11. International Atomic Energy Agency, Database of Prompt Gamma Rays from Slow Neutron Capture for Elemental Analysis, (2007)

12. S. Raman, et al., Phys. Rev. C, 46, 972, (1992)

13. A. M. Demidov, et al., Atlas of Gamma-Ray Spectra from the Inelastic Scattering of Reactor Fast Neutrons." Moscow, Atomizdat, (1978)

14. M. Herman, et al., Nuclear Data Sheets, 148, pp. 214-253 (2018) 